[This document contains the author's accepted manuscript. For the publisher's version, see the link in the header of this document.]

\title{
The Effect of Two Handwriting Approaches, D'Nealian and Sunform, on Kindergartners' Letter Formations
}

\author{
By Donita Massengill Shaw, Ph.D. \\ Associate Professor of Literacy Education
}

\author{
Paper citation: \\ Massengill Shaw, D. (2011). The effect of two handwriting approaches, D'Nealian and \\ Sunform on kindergartners' letter formations. Early Childhood Education \\ Journal, 39(2), 125-132. DOI 10.1007/s10643-011-0444-2.
}

Keywords:

handwriting, kindergarten, curriculum, legibility, alphabet

\begin{abstract}
:
The purpose of this study was to evaluate the effect of two handwriting approaches, D'Nealian and Sunform, on kindergartners' letter formations. Forty-one participants received D'Nealian handwriting instruction as the control group; 133 kindergartners were instructed in Sunform as the experimental approach. Pre-post tests at the beginning and end of school year asked kindergarten students to write the letters of the alphabet from memory. The letter formations were scored on a 4-point rubric for directionality and integration. The results showed the Sunform group had significantly higher scores on all but three letters of the alphabet. The D'Nealian students had considerably lower scores on missing or extra strokes, distortions and open letters. The findings of this study support the value of using an integrated, meaningful curriculum which appeals to young children and promotes a strong motor plan by requiring students to cross the midline to form counter-clockwise circles and diagonal lines. Implications for future research are included.
\end{abstract}


In recent years, handwriting instruction in elementary schools has been neglected due to numerous causes such as the use of technology, lack of time, inadequate teacher knowledge, and emphasis on standardized testing (Bennett, 2009; DuPree, 2009; Pressler, 2006; Suddath, 2009). So does handwriting instruction really matter? Is there value in teaching penmanship? Research states handwriting instruction is important for academic purposes (Graham, Berninger, Abbott, Abbott, \& Whitaker, 1997). It should be taught through a structured process that allows students to develop automaticity much like reading fluency (Graham, et al., 1997) because the ease and speed of writing impact a person's academic career and life. Sheffield (1996) stated teaching handwriting is a critical skill for three reasons: it allows access to kinesthetic memory, spontaneous handwriting allows students to concentrate on higher-level thinking and expression, and the world judges people based on their handwriting. Handwriting is too important to be left for chance learning; every child deserves the benefits of legible handwriting (Zaner-Bloser, 2004).

This article attempts to contribute to the research base by comparing D'Nealian handwriting with a new method known as Sunform taught to kindergartners in rural Mid-western school districts. D'Nealian is a handwriting system that minimizes formational differences between manuscript and cursive; letters are slightly oval and slanted with ending strokes. D'Nealian letters are abstract and may challenge students to remember distinct motor plans, which results in open, distorted and illegible letters. D'Nealian handwriting is taught separately from letter-sound instruction. In contrast, Sunform is a handwriting system that minimizes abstractness and reversals by teaching letters with a picture using counter-clockwise circles and strokes that require students to cross the midline and form distinct motor plans. Sunform handwriting is integrated with phonics instruction. Specifically we wanted to know: 
1. Were there differences between Sunform or D'Nealian students in total letter formation for all 26 letters?

2. Were there differences between the Sunform group and the D'Nealian group on letter formation for each letter of the alphabet?

3. Which letters of the alphabet were most difficult for kindergarten children to correctly form? Were the difficult letters the same for each of the two approaches or were there differences between approaches in which letters were difficult?

4. Which formational feature errors (correct start, directionality, integrated open, integrated close) were most common and were there differences between students of D'Nealian and students of Sunform in the type of feature errors they made?

\section{Literature Review}

The skills needed to write a letter of the alphabet may seem automatic to adults, but developmentally, writing the grapheme is challenging and requires concentration for young children. Students must retrieve the letter form and hold it in working memory, identify the letter's motor plan, establish the size of the letter, and execute/write it (Graham, Harris \& Fink, 2000). Students need to be able to control their fine motor abilities, possess eye-hand coordination, cross the midline, use a dominant hand and be able to hold a utensil (Marr, Windsor, \& Cermak, 2001).

Common errors are letter formations (57\%), reversals (52\%) and overall neatness (76\%) (Graham, Harris, Mason, Fink-Chorzempa, Moran \& Saddler, 2008). “Often children avoid analysis by starting at the base line for all letters and stroking away from their bodies" (Sheffield, 1996, p. 28). Some students do not retain in memory the kinesthetic pattern for forming letters so they need practice building repetition of motor plans. This kinesthetic memory is critical for 
success because it is the earliest, strongest and most reliable learning channel for young children (Sheffield, 1996). Many students who are referred for occupational therapy do not have dysfunctional skills; instead, they need structured and consistent instruction on letter formation (Asher, 2006).

Three instructional approaches include Zaner-Bloser, D'Nealian and Sunform. ZanerBloser (2004) has packaged materials known as a ball-and-stick or circle-and-stick approach. Often ball-and-stick is a favored approach because its font is thought to be similar to text young children read, although it has separate forms for manuscript and cursive. Children who learn ball-and-stick often separate their strokes and have reversals. In contrast, D'Nealian (Scott Foresman \& Co., 1978) was created to ease students' transition from manuscript to cursive by using letters that are similar between the forms; only five letters are shaped differently in the cursive alphabet than in the manuscript. The letters are slightly oval and slanted rather than rounded and vertical as in traditional ball/stick manuscript. Ending strokes are added to the manuscript letters and letters are formed continuously rather than with many stops/starts. Sunform (Sundberg, 1994) is an integrated handwriting and phonetic approach to learning sounds and letter formations that draws on the right hemisphere of the brain rather than the traditional left hemisphere of the brain. For each letter of the alphabet, the author designed one colored picture which represents the pure phoneme and allows for motorsensory movements. The picture name, sound and shape are integrated and meaningful rather than separated and disconnected. Reversals are diminished because children visualize the critical features of each picture as they draw/write it with continuous strokes starting at specific points and crossing the midline. Sunform differs from D'Nealian because Sunform letters are instructed initially through pictures and Sunform requires students to form a counter-clockwise circle and cross the 
midline, which prevents students from forming flat sided circles, open and distorted letters. For children who are neurologically challenged, Sunform provides an avenue for success (Massengill, Sundberg, \& Stewart, 2006).

Minimal research exists on kindergarten handwriting. Vander Hart, Fitzpatrick, and Cortesa (2009) observed four kindergarten teachers' literacy instruction. They found a lack of daily explicit handwriting instruction that paralleled Asher's (2006) findings. Teachers in this study did not, but should, ask children to write for fluency, from memory, and self-evaluate their handwriting. Vander Hart et al. suggested future research should evaluate reading/writing curriculums that complement each other, effectiveness of research-based strategies, impact of teacher training, and the development of an assessment tool. Handwriting curriculums have room for improvement. Kindergarten teachers need to evaluate available curricula to determine if they are motivational for young children and allow them to develop foundational handwriting skills (Marr, Windsor \& Cermak, 2001).

The assessment of penmanship is limited (Graham, 1986). Most handwriting programs outline how to teach the form, but lack a direct measure of whether the approach is effective. Neither do most programs provide a means to monitor progress, individualize instruction, or determine the competence of one's handwriting (Graham, 1986). In order to evaluate a letter's legibility, the assessor should make sure all parts of the letter are present, judge if any letter parts are rotated or reversed, and evaluate that each part is correctly formed (Graham, Weintraub, \& Berninger, 2001). Starting in the correct place and moving in the correct direction are two important criteria for letter formation (Nelson \& Trafford, 2007).

Graham (1986) evaluated the reliability, validity, and utility of three handwriting measures: a holistic rating system in which letters were rated on a 5 -point Likert scale $(1=$ 
illegible, 5 = well-formed letter), a holistic rating system with model letters, and a correctincorrect procedure. Results showed the two holistic scoring procedures, with and without model letters, were not reliable. However, the correct/incorrect measure was most consistent across raters and time satisfying the criteria of a relatively abstract construct for a quality such as letter formation. None of the three measures met all the criteria for reliability and validity sensitive to individual student differences and individual letters.

In sum, students must possess numerous skills, including motor plan/memory, in order to produce legible handwriting. Kindergartners need explicit instruction in letter formation through curricula that appeals to their developmental abilities and simultaneously supports mastery of letter sounds. Unfortunately, there are few published handwriting assessments that meet criteria for reliability and validity. Assessors should judge if letters are formed correctly without reversals or rotations, and all parts of the letter are present. This study addresses gaps in the literature by evaluating kindergarten handwriting curriculum that builds students' motor plan/memory in a developmentally appropriate way. Further, an assessment tool is provided in this manuscript.

\section{Methodology}

\section{Participants}

Control. Control, also known as D'Nealian, participants were kindergarten students enrolled in a rural school district in a Midwestern state. Of the 41 control participants, $95 \%$ were Caucasian and 5\% were African American. None were English language learners. There were fewer males (41\%) than females (59\%). Fifty-one percent of the kindergartners received free or reduced lunch. 
Two female teachers had six years of teaching experience and the third kindergarten teacher was a veteran of 35 years. None of the kindergarten teachers obtained master degrees.

Experimental. Experimental, also known as Sunform, participants were kindergarten students enrolled in a school district in a neighboring town which was the county seat. There were a total of 133; approximately half were boys (49\%) and half were girls (51\%). Most were Caucasian (77\%). Thirteen percent were African American, 2\% Native American, and 6\% were Latino. Ten of the total kindergartners were English Language Learners who received additional support. Sixty-two percent of the students received free or reduced lunch.

There were seven experimental teachers ranging in experience from 3 years to 32 years. The average teaching career was 21 years. Only one teacher was male. Five teachers held master degrees.

\section{Classrooms and Fidelity}

The two neighboring school districts differed in size and curriculum. The control/D'Nealian district had fewer kindergarten classrooms $(\mathrm{N}=3)$ and a lower teacher/student ratio (1:18) than the experimental/Sunform classrooms $(\mathrm{N}=7)$ and teacher/student ratio (1:22). Teachers at each school were expected to teach the assigned D'Nealian or Sunform curriculum and had received an inservice on teaching handwriting. All classrooms had handwriting charts of the respective system posted on classroom walls for students to use. Teachers at both districts incorporated writing through journaling, rhyming words, word families, and sentence prompts. Writing activities supported handwriting practice.

Teacher fidelity was measured through observations by three personnel: the principal, reading specialist/learning coach, and a trained assessor a minimum of three times per year: fall, winter and spring. Documented notes of what the teachers did in their classrooms during 
observations were compared among the three personnel for triangulation. The fidelity for the assigned teaching method was good or very good among all the teachers. Although there were stylistic teaching differences, it was confirmed that the experimental teachers implemented the Sunform method with consistency and the control teachers thoroughly taught D'Nealian.

\section{Procedures}

\section{Instructional procedures}

Control. The control students spent 90-120 minutes each morning involved in literacy tasks using the reading program A Legacy of Literacy published by Houghton-Mifflin and the handwriting program D'Nealian. When the teachers introduced alphabet letters to the whole class in traditional a-z order, they modeled how to form the letter using D'Nealian style on the chalkboard. After modeling a letter, the children were asked to practice writing the letter on lined worksheets. This whole group instruction occurred for approximately 10-15 minutes each day. Small-group follow-up instruction was given to students who needed extra assistance with letter formation. Learning centers such as handwriting practice in various formats (sand, etc.) were utilized.

Experimental. Students in the experimental classrooms also spent 90-120 minutes each morning on literacy using the reading program Treasures by McGraw Hill. The teachers incorporated both reading and handwriting using whole and small group instruction and learning centers. The Sunform handwriting instruction categorized the 26 letters into six groups based on motor plan. Students first identified a key picture and produced a pure phoneme, then transitioned to visual clues and finally learned the graphemes. Handwriting instruction was taught through whole group instruction for approximately 10-15 minutes a day. Students independently completed workbook pages after learning how to form each letter. Small groups 
were used for children who needed extra assistance. Handwriting centers, such as practicing letters in sand, were used each week.

\section{Testing procedures}

All control and experimental students were individually pre-tested in August and posttested in May. Pre and post-testing procedures were identical at both sites. A trained and qualified tester provided a lined sheet of paper for each child and asked the child to write the lower case alphabet in the aforementioned order based on motor plan, not traditional alphabetical order. All children were asked to write the letters from memory as recommended by Graham, Weintraub, and Berninger (2001) for ecological validity. When finished with each letter the tester marked any errors such as incorrect starting place or writing in the wrong direction (i.e. bottom to top). If a child took longer than five seconds to respond, the tester asked the child to write the next grapheme. The testing of each individual student took approximately ten minutes.

\section{Coding procedures}

Prior to this research study, the Sunform author went through hundreds of student samples and identified 26 motor plan error types that could occur, although error differentiation depended on each specific letter. The broad letter formation error categories included spatial, cross midline, directionality, integration/close, integration/open and distortions. These are reported in a previous study (Massengill, Sundberg, \& Stewart, 2006).

For this kindergarten study, four dominant categories were identified from the 26 motor plan errors and a 4-point rubric scale was created based on the correct/incorrect model (Graham, 1986). Two points were awarded for directionality and two points for integration.

Directionality points were given if the child started the letter in the correct place and moved in the correct direction (e.g. right to left, top to bottom) with no reversals. Another two points were 
given for integration which means the letter was closed, no extra strokes were added, there were no missing strokes and the letter was recognizable as the intended letter. Because kindergartners often struggle with size and proportion, we did not deduct points if the letter went above or below the allotted lines. After all the pre-post tests were administered, four knowledgeable and qualified assessors were hired to score both the control and experimental data based on the 4point rubric. Inter-rater reliability was $>.90$.

\section{Data analysis}

All information was entered in a statistical program. Each student's row of data included individual letter scores pre-post, rubric scores, and an overall total score for the pre-post test. Individual letter scores were 1-4 points; each of the 26 letters of the alphabet could receive up to 4 points based on the grading rubric. This 1-4 number was listed in a column for each of the 26 letters. Rubric scores followed each individual letter. Students could receive either a 0 (incorrect) or 1 (correct) for each feature of the rubric $(\mathrm{c}=$ correct start, $\mathrm{d}=$ directionality, ic $=$ integrated closed, io $=$ integrated open). For example the spreadsheet had a 1-4 score for the individual letter $\mathrm{v}$ in one column, followed by four columns with a score of 0 or 1 : vc (letter $\mathrm{v}$ correct start), vd (letter $\mathrm{v}$ directionality), vic (letter $\mathrm{v}$ integrated close), vio (letter $\mathrm{v}$ integrated open). In this way we could see how the students formed each letter of the alphabet before and after intervention. Finally we entered a total overall score. The highest total score possible was 104 points, which came from awarding up to 4 points per letter. A number of statistical analyses were conducted and are described in the results. 


\section{Results}

The results will be reported by the research questions.

Were there differences between Sunform or D'Nealian students in total letter formation for all 26 letters?

We used analysis of covariance to test group differences on students' total letter formation scores (total $=104$ points) using their pre-test scores as the covariate. In this way we accounted for pre-instructional group differences, a statistical way of leveling the playing field by factoring in skills some children may have had before the start of the school year. After accounting for pre-instructional differences, the Sunform group $(M=9.70 ; S D=10.37)$ outperformed the D'Nealian group $(M=8.48 ; S D=10.35), F(1,170)=119.75, p<.001$. The Sunform group performed $57.9 \%$ better than the D’Nealian group.

These results show that the Sunform participants significantly improved more than the participants who were taught with the D'Nealian method. The teachers and researchers attempted to parallel the amount of instructional time both groups received on handwriting. This would indicate that the amount of growth of kindergartners in the experimental group could not have occurred with the D'Nealian approach.

Were there differences between the Sunform group and the D'Nealian group on letter formation for each letter of the alphabet?

We used a multivariate analysis of variance to test group differences on letter formation scores (1-4 points) for all of the letters taken together. There was a significant difference between the two groups taking all the letter scores together, Wilks $\Lambda=.455, \mathrm{~F}(26,145)=6.69$, $\mathrm{p}<, 001, \eta^{2}=.54$. Since this omnibus test was significant, univariate follow-up tests were conducted; the criterion for significance (alpha) was adjusted to .001 using a Bonferroni 
adjustment, a common statistical correction when making multiple pairwise comparisons. We found there were differences and that the Sunform group had significantly higher scores than the D'Nealian on 23 letters. The groups were not different from each other for the letters $v, p, s$. Which letters of the alphabet were most difficult for kindergarten children to correctly form? Were the difficult letters the same for each of the two approaches or were there differences in which letters were difficult among approaches?

We ordered the post-test letters by rank (easiest to hardest) according to mean. As can be seen in Table 1, $v$ was the letter with the highest mean for both methods, which indicates it was the easiest to form. On a 4-point scale, $v$ received a mean of 3.66 for the Sunform students and 3.07 for the D'Nealian students. Letter $p$ contained the most errors by the Sunform students and was the most difficult to form, but the mean differences between the two groups were not dissimilar ( $M=2.41$ experimental; $M=2.30$ control). When looking at the mean scores, 20 out of the 26 letters formed by Sunform students had means above the highest mean for the D’Nealian students $(M=3.07)$. See Table 1 .

We trichotomized the 26 letters into three groups by rank: easy, medium, hard. The nine top ranked letters were easy to form, the eight middle letters posed medium challenge, and the nine lowest ranked letters were the most difficult to form. Four out of the nine letters $v, i, e, u$, were easy for both Sunform and D'Nealian students. Letters $a, b, g, n$ were medium difficulty for both approaches. The six most challenging letters for both groups were $f, q, k, m, d, l$. 
Which formational feature errors (correct start, directionality, integrated open, integrated close) were most common and were there differences between students of D'Nealian and students of Sunform in the type of feature errors they made?

A repeated measures analysis of variance with one within-subjects factor (feature) and one between-subjects factor (school-method) was used to test whether there were differences in the frequencies of different formational feature errors and to test whether there were differences between Sunform and D'Nealian in the types of errors they made. There was a significant effect of feature error, indicating that at least one type of error was significantly more frequent than at least one other feature error, Wilks $\Lambda=.244, \mathrm{~F}(3,169)=174.4, \mathrm{p}<.001, \eta^{2}=.756$. There was also a significant interaction of feature error with instructional method, Wilks $\Lambda=.526, \mathrm{~F}$ (3, 169) $=50.84, \mathrm{p}<.001, \eta^{2}=.474$.

The significant interaction between feature error and instructional method means that students in the D'Nealian group produced a different pattern of feature errors than the Sunform students. We examined these differences by analyzing the groups separately using a repeated measures analysis of variance with one within-subjects factor (feature) to test feature differences. For the Sunform group there was a significant feature effect, Wilks $\Lambda=.396, \mathrm{~F}(3,130)=$ $66.134, \mathrm{p}<.001, \eta^{2}=.604$. Follow-up pairwise comparisons with a Bonferroni adjustment for multiple comparisons found each feature was significantly different from every other feature. Likewise, for the D'Nealian group there was a significant feature effect, Wilks $\Lambda=.093$, F (3, $37)=119.6, p<.001, \eta^{2}=.91$, and follow-up pairwise comparisons, adjusted for multiple contrast, found each feature was significantly different from every other feature.

Table 2 shows the mean feature score for each instructional method. According to the rubric students received one point for each correct feature so higher means indicate more 
students formed that feature correctly. In both methods the Integrated Close feature was highest; it was easiest for students to make letters approximate the appearance of the model letter. The mean for the feature Integrated Open (missing/extra strokes or parts, distortions, open letters) was significantly lower for the D'Nealian students than for the Sunform.

\section{Conclusion}

This study investigated the letter formations of kindergartners enrolled in schools with two different handwriting approaches: D'Nealian and Sunform. When looking at letter formation points obtained for all 26 letters, we learned that the Sunform students had higher scores on their letter formation, which may have resulted for two reasons. First, Sunform students identified a picture before they learned the letter's name, sound and motor plan. Most children initially find arbitrary abstract symbols (i.e. letters) to be meaningless. It is to the students' advantage to learn the linear abstract alphabet through mnemonic imagery (Adams, 1990), emotion and movement (Hannaford, 1995) and active exploration of the relationships between sounds, visual characteristics and motor movement of letter formation (Bear, Invernizzi, Templeton \& Johnston, 2008). This new integrated alphabet system was able to assist students to use their imagination to link an abstract symbol to known information. Another reason these students were able to successfully learn their letter formations was due to the fact this approach integrates the visual (e.g. picture of a cat), auditory (e.g. alliteration of the "cat catching") and the kinesthetic (e.g. motor plan). Sylwester (1995) wrote,

When objects are registered by several senses, they can be stored in several interrelated memory networks. A memory stored in this way becomes more accessible and powerful than a memory stored in just one sensory area, because each sensory memory checks and extends the others (p. 96). 
Traditional methods of teaching sounds and letter formations rely on detail and rote memorization, and often phonics (sound learning) is separated from handwriting (grapheme learning). For example, when students wrote in D'Nealian they did not say the sound of the letter. In contrast, the integrated Sunform method appealed to students' visual ability to stimulate auditory recall of letter sounds and proper formation. In other words, the Sunform students pronounced the sound as they wrote the letter. The integrated alphabet system builds on multisensory learning by integrating the visual, auditory and motor learning so the three cannot be separated. It is this integration of learning that makes the Sunform approach intersensory.

The second research question investigated the differences of the two groups by looking at each individual letter score rather than total score. We found significant differences on all but three letters, $v, p$, $s$. For both approaches, ' $\mathrm{v}$ ' was the highest ranked letter, indicating it was the easiest to form. Starting at the top left, angling toward the bottom line and then returning to the top line makes ' $\mathrm{v}$ ' one of the easiest for students regardless of the approach because it does not challenge the midline. There are few possibilities for missing or added strokes and the letter is already open and can't be reversed. Letter 'p' provided the most challenges for the Sunform students. The Sunform author attributes the difficulty to the picture; her original picture idea was abandoned and she has continually seen students' formation problems with the published letter $p$ (i.e. plumbing pipe). This finding indicates how the critical features of a picture impacts students' formations. Although Sunform students had the most challenge with letter $\mathrm{p}$, the mean differences between groups were not significant. The letter $s$ ranked in the top tier for D'Nealian students and ranked in the lowest tier for Sunform students, yet the Sunform mean was higher ( $M$ $=2.78$ D'Nealian; $M=3.01$ Sunform) and they were not significantly different. One possible reason for difference in rank was related to all the other letters of the alphabet. A continuous line 
that started at the top and went to the bottom was simple for the D'Nealian students comparatively. In contrast, the Sunform students were successful at many other letters with greater challenges (i.e. $k, q, r, b$ ), so they did not give the formation of $s$ as much thought or effort and a lower score resulted.

Research question three investigated the rank order shown in Table 1. We discovered about half of the letters in each tier (easy, medium, hard) ranked similarly for both approaches. This indicates some likeness across instructional method; for example, letter $u$ is easy, letter $g$ is harder and letter $k$ is one of the most difficult for children to form. This finding is consistent with previous research that letters vary in their difficulty (Graham, et al., 2001). No other study has evaluated kindergartners' letter formations. Graham, Weintraub, and Berninger (2001) found the most legible letters for first graders who used traditional manuscript were $s, e, c$. Letters with some difficulty were $o, v, x, i, m, w, p, f, r$. The most difficult letters were $q, z, g, u$, $n, k, j, y$. Because first grade is closest to kindergarten, we looked to see if any of the easy and difficult letters were comparable among the previous and current research study. We discovered minimal overlap in results: letter $e$ was easy, and $q$ and $k$ were difficult across methods and grade levels.

When comparing each letter of the alphabet method to method, Sunform students had higher means on all of the letters. The results could be attributed to two possibilities: either the instruction or the method. We looked to see if there were differences in students' scores by classroom teacher within each school and we found considerable variation, which was also confirmed in the teacher fidelity observations. Although groups of students have different needs and abilities, most likely the differences in scores may also be attributed to teacher variation in instructional explicitness, daily practice, and amount of emphasis given to handwriting. No 
matter the curriculum, teaching differences are to be anticipated. We assert the higher letter means for Sunform students were a direct result of method. The Sunform picture appeals to 5year old children, is retained in memory, and promotes a strong motor plan that crosses the midline and requires counter-clockwise circles. These features cannot be underestimated.

For the fourth research question we found differences among features for both instructional methods. This confirms that all four features identified in the rubric are important to letter formation. We also learned that the D'Nealian students struggled more with all four features, but especially with adding extra strokes, forming letters with missing strokes, and distorting the letters. Teachers who taught using traditional ball-and-stick or D'Nealian reported most of their students' errors resulted from letter formation (57\%), reversals (52\%) and overall neatness (76\%) (Graham, et al., 2008). In contrast, the results of this study show Sunform students have significantly reduced errors.

Graham, Weintraub \& Berninger (2001) identified six characteristics of letter legibility, but found five to be significant: no breaks, no additional lines/strokes, correctly proportioned, correctly formed, no reversals or rotated parts. In our study we embedded these features into our rubric. For example, no breaks and no additional lines/strokes were Integration Open. Reversals or rotated parts were scored as Directionality. We did not score proportion because we recognize that some kindergarten children may struggle developmentally with the top/bottom lines and our focus was on correctly forming the letters. Finally, the students in this research study could receive 4 out of 4 for each letter, indicating it was formed correctly.

The rubric developed and presented in this study was shown to be reliable among raters as previously mentioned. We assert that this rubric has value for classroom teachers and researchers; it is easy to use because a teacher can quickly determine if the feature is present. 
Further, support from Graham (1986) identified that of the three measures, only the correct/incorrect rating scale appeared to satisfy two criteria: "different raters using the same measure and a single rater using the same measure on different occasions" (p. 379). Future research does need to identify whether the rubric developed for this study possesses all the essential psychometric properties.

Classroom observations using a structured fidelity measure (high, medium, and low) and teacher interviews should be embedded in future research. A fidelity measure would add reliability to the instructional method and allow researchers to conduct regression analyses. Further, interviews would allow the researcher to tap into teachers' belief systems about the importance of teaching handwriting. Another limitation is that we did not measure the speed in which students wrote their letters, nor did we ask the kindergartners to write sentences or construct stories. Further inquiry should include these ideas to triangulate letter formations and handwriting legibility on multiple tasks. Future research should also compare the two handwriting approaches for differences in achievement among boys and girls (Graham, 1986) and those referred to occupational therapists (Asher, 2006) or who are considered poor handwriters (Graham, Struck, Santoro, \& Berninger, 2006). Another possibility is to use more than one measure including standardized testing or assessments used by Graham and colleagues (Graham, 1986; Graham et al., 2001, 2006).

In sum we recognize limitations of this study, yet we also see the value of teaching students to form letters correctly by using the Sunform developmental approach. We have evidence across several studies that the characteristics embedded into the Sunform approach produce children who can correctly form their letters (Massengill, Sundberg \& Stewart, 2006; Massengill Shaw \& Sundberg, 2008). We have identified curriculum that is motivational for 
young children and simultaneously provides foundational skills (Marr, Windsor, \& Cermak, 2001). This study has also contributed to the field by identifying effective strategies and features in a research-based curriculum as recommended by Vander Hart, Fitzpatrick, \& Cortessa (2009). Handwriting is a critical beginning for later writing fluency. By preventing difficulties in the early grades we can assist students to be successful in communicating their ideas and thoughts (Graham et al., 1997; Graham, et al., 2000; Sheffield, 1996). 


\section{$\underline{\text { References }}$}

Adams, M. J. (1990). Beginning to read, thinking and learning about print. Cambridge, MA: MIT Press.

Asher, A. V. (2006). Handwriting instruction in elementary schools. The American Journal of Occupational Therapy, 60(4), 461-471.

Bear, D. R., Invernizzi, M., Templeton, S., \& Johnston, F. (2008). Words their way. Upper Saddle River, NJ: Merrill.

Bennett, J. (2009). The curse of cursive. Newsweek, February 23, 2009.

DuPree, W. (2009). Teachers afraid cursive might become a lost art. Lawrence Journal World, November 7, 2009.

Graham, S. (1986). The reliability, validity, and utility of three handwriting measurement procedures. Journal of Educational Research, 79, 373-380.

Graham, S., Berninger, V., Abbott, R., Abbott, S., \& Whitaker, D. (1997). Role of mechanics in composing of elementary school students: A new methodological approach. Journal of Educational Psychology, 89, 170-182.

Graham, S., Harris, K. R., \& Fink, B. (2000). Is handwriting causally related to learning to write? Treatment of handwriting problems in beginning writers. Journal of Educational Psychology, 92(4), 620-633.

Graham, S., Harris, K. R., Mason, L., Fink-Chorzempa, B., Moran, S., \& Saddler, B. (2008). How do primary grade teachers teach handwriting? A national survey. Reading and Writing: An Interdisciplinary Journal, 21(1-2), 49-69.

Graham, S., Struck, M., Santoro, J., \& Berninger, V. W. (2006). Dimensions of good and poor handwriting legibility in first and second graders: Motor programs, visual-spatial 
arrangement, and letter formation parameter setting. Developmental Neuropsychology, 29(1), 43-60.

Graham, S., Weintraub, N., Berninger, V. (2001). Which manuscript letters do primary grade children write legibly? Journal of Educational Psychology, 39(3), 488-497.

Hannaford, C. (1995). Smart moves. Arlington, VA: Great Ocean Publishers.

Marr, D., Windsor, M. M., \& Cermak, S. (2001). Handwriting readiness: Locatives and visuomotor skills in the kindergarten year. Early childhood research and practice, 3(1). Retrieved March 22, 2004 from http://ecrp.uiuc.edu/v3n1/marr.html

Massengill, D., Sundberg, M .L., \& Stewart, A. (2006). A unique, neurologically integrated approach designed to teach letter sounds and formations. Reading Improvement, 43(3), $111-128$.

Massengill Shaw, D., \& Sundberg, M .L. (2008). How a neurologically integrated approach which teaches sound-symbol correspondence and legible letter formations impacts at-risk first graders. Journal of At-Risk Issues, 14(1), 13-21.

Nelson, R. H, \& Trafford, C .H. (2007). Evidence based handwriting instruction self evaluation guide. Peterson Directed Handwriting. Retrieved September 3, 2009 from Www.peterson-handwriting.com

Pressler, M. W. (2006). Except for a few defenders, cursive all but written off. Lawrence Journal World, October 12, 2006.

Scott Foresman \& Company. (1978). D’Nealian manuscript alphabet. Chicago, IL.

Sheffield, B. (1996). Handwriting: A neglected cornerstone of literacy. Annals of Dyslexia, 46, 21-35. 
Suddath, C. (2009). Mourning the death of handwriting. Time, August 3, 2009. Retrieved August 3, 2009 from www.time.com

Sundberg, M. L. (1994). Sunform Alphabet System Manual. Lake Bluff, IL: Sundberg Learning Systems, Inc.

Sylwester, R. (1995). A celebration of neurons: An educator's guide to the human brain. Alexandria, VA: Association for Supervision and Curriculum Development.

Vander Hart, N., Fitzpatrick, P., \& Cortesa, C. (2009). In-depth analysis of handwriting curriculum and instruction in four kindergarten classrooms. Reading and Writing (online edition) April 20, 2009. Retrieved September 30, 2009.

Zaner-Bloser (2004). How legible handwriting enhances the writing process and assessment. Retrieved March 22, 2004 from www.zaner-bloser.com 
Table 1

Means for each letter of the alphabet on the post-test*

\begin{tabular}{|c|c|c|c|}
\hline Sunform letter order & Mean & D’Nealian letter order & Mean \\
\hline$\overline{\mathrm{V}}$ & 3.66 & $\mathrm{~V}$ & 3.07 \\
\hline I & 3.62 & $\mathrm{U}$ & 2.90 \\
\hline $\mathrm{W}$ & 3.59 & S & 2.78 \\
\hline $\mathrm{Y}$ & 3.55 & I & 2.67 \\
\hline $\mathrm{E}$ & 3.54 & $\mathrm{E}$ & 2.65 \\
\hline $\mathrm{U}$ & 3.53 & $\mathrm{X}$ & 2.58 \\
\hline $\mathrm{H}$ & 3.51 & $\mathrm{C}$ & 2.52 \\
\hline $\mathrm{T}$ & 3.39 & $\mathrm{O}$ & 2.50 \\
\hline $\mathrm{R}$ & 3.36 & $\mathrm{Z}$ & 2.35 \\
\hline $\mathrm{Z}$ & 3.33 & $\mathrm{P}$ & 2.30 \\
\hline$X$ & 3.29 & $\mathrm{R}$ & 2.25 \\
\hline $\mathrm{C}$ & 3.29 & $\mathrm{~J}$ & 2.25 \\
\hline $\mathrm{G}$ & 3.27 & $\mathrm{G}$ & 2.25 \\
\hline $\mathrm{O}$ & 3.26 & $\mathrm{~N}$ & 2.20 \\
\hline A & 3.18 & $\mathrm{~B}$ & 2.15 \\
\hline $\mathrm{N}$ & 3.15 & A & 2.15 \\
\hline B & 3.14 & $\mathrm{H}$ & 2.15 \\
\hline $\mathrm{L}$ & 3.13 & $\mathrm{~L}$ & 2.10 \\
\hline Q & 3.09 & W & 2.10 \\
\hline $\mathrm{J}$ & 3.07 & $\mathrm{~T}$ & 2.05 \\
\hline $\mathrm{K}$ & 3.01 & $\mathrm{Y}$ & 1.93 \\
\hline$S$ & 3.01 & $\mathrm{M}$ & 1.83 \\
\hline $\mathrm{F}$ & 2.97 & $\mathrm{Q}$ & 1.70 \\
\hline $\mathrm{M}$ & 2.85 & $\mathrm{~F}$ & 1.58 \\
\hline $\mathrm{D}$ & 2.50 & $\mathrm{D}$ & 1.58 \\
\hline $\mathrm{P}$ & 2.41 & $\mathrm{~K}$ & 1.35 \\
\hline
\end{tabular}

The first 9 letters are considered easy to form

The middle 8 letters are considered of medium challenge to form

The lowest 9 letters are considered difficult to form

*Scores were based on a 4-point scale 
Table 2

Mean Feature Score for Each Instructional Method

\begin{tabular}{lcc} 
Rubric feature & Sunform mean & D'Nealian mean \\
\hline $\mathrm{C}=$ correct start & .705 & .495 \\
$\mathrm{D}=$ directionality & .832 & .630 \\
$\mathrm{IC}=$ integrated closed & .909 & .784 \\
$\mathrm{IO}=$ integrated open & .772 & .338 \\
\hline
\end{tabular}

